\begin{tabular}{|c|l|}
\hline Title & Mayer-V ietoris sequences and coverage problems in sensor networks \\
\hline Author(s) & A rai, Zin; Hay ashi, Kazunori; Hiraoka, Y asuaki \\
\hline Citation & $\begin{array}{l}\text { Japan Journal of Industrial and A pplied Mathematics, 28(2), 237-250 } \\
\text { https://doi.org/L0.1007/313160-011-0039-8 }\end{array}$ \\
\hline Issue Date & 2011-08 \\
\hline Doc URL & http://hdl.handle.net/2115/47244 \\
\hline Rights & The original publication is available at www.springerlink.com \\
\hline Type & article (author version) \\
\hline File Information & JJA M28_2_237-250.pdf \\
\hline
\end{tabular}

Instructions for use 


\title{
Mayer-Vietoris sequences and coverage problems in sensor networks
}

\author{
Zin Arai · Kazunori Hayashi · \\ Yasuaki Hiraoka
}

\begin{abstract}
A coverage problem of sensor networks is studied. Following recent works by Ghrist et al., in which computational topological methods are applied for the coverage problem, We present an algorithm for the distributed computation of the first homology group of planar Rips complexes. The key idea is to decompose a Rips complex into smaller pieces of subcomplexes, and to make use of Mayer-Vietoris sequences in order to sum up the homology groups of subcomplexes. Combined with a sufficient condition for the coverage which is given in terms of the first homology group, the proposed algorithm enables us to verify the coverage in a distributed manner.
\end{abstract}

Keywords Mayer-Vietoris sequences · sensor networks · coverage problems

\section{Introduction}

A wireless sensor network, which consists of a number of sensor nodes with signal processing and communication capabilities, has been drawing a lot of research interest since its birth in the early nineties. What make the sensor network different from conventional broadband wireless communications systems are crucial requirements for the sensor nodes of low cost and high energy

Z. Arai

Creative Research Institution, Hokkaido University / JST PRESTO

N21W10, Kita-ku, Sapporo 001-0021, JAPAN

E-mail: arai@cris.hokudai.ac.jp

K. Hayashi

Graduate School of Informatics, Kyoto University

Yoshida-Honmachi, Sakyo-ku, Kyoto, 606-8501, JAPAN

E-mail: kazunori@i.kyoto-u.ac.jp

Y. Hiraoka

Graduate School of Science, Hiroshima University / JST PRESTO

1-7-1, Kagamiyama, Higashi-Hiroshima, 739-8521, JAPAN

E-mail: hiraok@hiroshima-u.ac.jp 
efficiency, since a large number of battery-operated sensor nodes have to be utilized in common applications. Major power-intensive operations in sensor nodes are radio communication and signal processing [15]. Therefore the communications range should be strictly restricted, and reducing the amount of signal processing in sensor nodes is of great importance in the design of wireless sensor networks.

One of the fundamental problems in the wireless sensor network is a coverage problem. This is because the coverage problem is closely related to both the network deployment problem, which is directly related to the cost of the network, and the scheduling problem of sleep and wake-up modes for low power consumption. Moreover, the coverage is also regarded as one of the important measures for the quality of service provided by the wireless sensor network.

For the coverage problem in sensor networks, Ghrist et al. have recently presented computational topological methods ([3], [4] and [5]). Their idea is to capture global information about the covering from homology groups of a certain geometric object which can be constructed from local connection information between sensors. One of the advantages of a topological method is that it does not assume the information of absolute locations or orientations for the sensors, i.e., the method is coordinate-free. Moreover, there is no probabilistic assumption such as a uniform distributions of sensors in a given domain. These assumptions, which are sometimes regarded as unrealistic, are necessary for conventional techniques based on computational geometry (e.g.[7], [10], [12], [19]) or probabilistic approaches (e.g. [9], [16], [18]). Therefore, the topological methods have potentials for various practical applications of sensor networks.

Following the paper [3], let us here describe the settings of the coverage problem we treat.

Assumptions.

A1. Suppose that $P$ is a finite set of sensors (or also called nodes) in a compact connected domain $D \subset \mathbb{R}^{2}$.

A2. Two sensors $v, w \in P$ can communicate with each other when their distance is less than broadcast radius $r_{b}$.

A3. Each sensor has radially symmetric covering domains of cover radius $r_{c} \geq r_{b} / \sqrt{3}$.

A4. The boundary $\partial D$ is assumed to be connected and piecewise-linear with nodes marked fence nodes. The distance between any adjacent two fence nodes is less than $r_{b}$.

Let $B\left(v ; r_{c}\right)$ be a disk with radius $r_{c}$ centered at $v \in P$ and let $U=$ $\bigcup_{v \in P} B\left(v ; r_{c}\right)$. Then the coverage problem is to ask whether $U$ contains $D$. In this setting, the essential step of the topological methods is to build up a Rips complex [6][17], which can be constructed by local connection information determined by $r_{b}$ and possesses reasonable covering information $U$. Here, the Rips complex $\mathcal{R}_{r_{b}}$ of $P$ is an abstract simplicial complex and is defined as follows. All nodes in $P$ are assigned to be 0 -simplexes, and a $(k+1)$-tuple of nodes $v_{0}, \cdots, v_{k}$ determines a $k$-simplex $\left|v_{0} \cdots v_{k}\right|$ in $\mathcal{R}_{r_{b}}$ if and only if any two nodes of them are within the distance $r_{b}$. In this paper, we abbreviate 
$\mathcal{R}_{r_{b}}$ to $\mathcal{R}$, and treat their homology groups $H_{*}(\mathcal{R})$ as integer coefficients (see e.g.[14] for standard homology theory).

Let us note that, from $\mathbf{A} \mathbf{4}$, the boundary $\partial D$ can be identified with a subcomplex $\mathcal{F}$ of $\mathcal{R}$. Then, in the above setting, de Silva and Ghrist presented the following sufficient condition for the coverage $D \subset U$ :

Theorem 1 (de Silva and Ghrist [3]) The sensor covering $U$ contains $D$ if there exists $[\alpha] \in H_{2}(\mathcal{R}, \mathcal{F})$ such that $\delta_{2}[\alpha] \neq 0$ in $H_{1}(\mathcal{F})$, where $\delta_{2}$ is the connecting homomorphism arising in the long exact sequence of the pair $(\mathcal{R}, \mathcal{F})$ :

$$
\cdots \longrightarrow H_{2}(\mathcal{F}) \longrightarrow H_{2}(\mathcal{R}) \longrightarrow H_{2}(\mathcal{R}, \mathcal{F}) \stackrel{\delta_{2}}{\longrightarrow} H_{1}(\mathcal{F}) \longrightarrow \cdots
$$

The most important point of this theorem is, as we have already mentioned, that it does not require absolute coordinates of the sensors or probabilistic assumptions. The only necessary information is a graph structure for the set of nodes $P$, which is equivalent to the 1 -skeleton of $\mathcal{R}$ and is available from the conditions of communications between sensors.

Theorem 1 itself is a well worthy result in the sense of providing a novel method which can deal with difficulties for conventional methods, however, it includes several problems, especially for implementations, as mentioned in [3]. The most crucial problem among them is that, although the construction of the Rips complex only requires the local connection information, a naive computation of homology groups needs centralized processing. It should be avoided in the following senses.

First, it is not desirable for all sensors to transmit local connection information to a central base station, because this procedure requires huge amount of communications or long distance communications, and is, after all, equivalent to assuming the global information, like computational geometric approaches. Due to the restriction of batteries for sensors, such communications should not be adopted. Second, even if one can obtain the integrated connection information at one place, the present computational complexity of homology groups is in the range of orders from quadratic to quintic in the number of simplices [8], and we can not ignore this cost for practical implementations.

One possibility to overcome this problem is to adopt a distributed way of homology computations. That is to say, we would like to somehow sum up homology groups of small pieces of subregions in parallel in order to obtain the global homology group. This is the central subject to discuss in this paper.

Our main result in this paper is to present a distributed algorithm for checking the sufficient condition for the coverage under the assumptions A1A5 ("non-pinching condition" A5 and its verifiable substitution $\widetilde{\mathbf{A 5}}$ will be explained in section 3). The technique is based on distributed homology computations for $H_{1}(\mathcal{R})$, and deeply relies on Mayer-Vietoris sequences (see [14], for example), which are classical tools in algebraic topology to calculate a homology group of a union of two appropriate topological objects. Our algorithm 
consists of two parts. One is to derive a decomposition of a given Rips complex $\mathcal{R}=\cup_{k=1}^{K} \mathcal{R}_{k}$ (Algorithm 6), and the other is to sum up homology groups $H_{1}\left(\mathcal{R}_{k}\right)$ to $H_{1}(\mathcal{R})$ (Algorithm 8 ).

We here note that it is not trivial whether we can always calculate $H_{1}\left(\mathcal{R}_{1} \cup\right.$ $\mathcal{R}_{2}$ ) by the corresponding Mayer-Vietoris sequence, since this exact sequence only gives relationships among $H_{*}\left(\mathcal{R}_{1}\right), H_{*}\left(\mathcal{R}_{2}\right), H_{*}\left(\mathcal{R}_{1} \cap \mathcal{R}_{2}\right)$, and $H_{*}\left(\mathcal{R}_{1} \cup\right.$ $\left.\mathcal{R}_{2}\right)$. The key for the computability of $H_{1}\left(\mathcal{R}_{1} \cup \mathcal{R}_{2}\right)$, which is shown in Proposition 3, results from the following geometric good property of a planar Rips complex.

Suppose $p: \mathcal{R} \rightarrow \mathbb{R}^{2}$ is a projection map which maps each simplex in $\mathcal{R}$ affinely onto the convex hull of its nodes in $\mathbb{R}^{2}$. The projection of $\mathcal{R}$ is called the shadow and denoted by $S$. Let $\pi_{1}(\mathcal{R})$ and $\pi_{1}(S)$ be the fundamental groups whose base points are naturally identified by $p$. The projection map $p$ induces a homomorphism $\pi(p): \pi_{1}(\mathcal{R}) \rightarrow \pi_{1}(S)$ on these fundamental groups. Then, the following theorem holds:

Theorem 2 (Chambers, de Silva, Erickson and Ghrist [1]) Let $\mathcal{R}$ be a Rips complex generated by a finite number of points in $\mathbb{R}^{2}$. Then $\pi(p): \pi_{1}(\mathcal{R}) \rightarrow$ $\pi_{1}(S)$ is an isomorphism. Therefore, $H_{1}(\mathcal{R})=H_{1}(S)$ and hence $H_{1}(\mathcal{R})$ is free.

Let us also note the relationship between the sufficient condition for the coverage in Theorem 1 and the first homology group. Due to the help of Theorem 2 again and the non-pinching assumption A5 or $\widetilde{\mathbf{A 5}}$ with slight modifications of fence nodes, Proposition 4 below states that studying $H_{1}(\mathcal{R})$ is enough to check the sufficient condition in Theorem 1. That is, we can show that under the assumptions A1-A5 (or $\widetilde{\mathbf{A 5}}$ ), sensors cover $D$ if $H_{1}(\mathcal{R})$ is vanishing (Corollary 5).

To solve the coverage problem, therefore, it suffices to construct an efficient algorithm for computing $H_{1}(\mathcal{R})$. For this purpose, we are going to present Algorithm 8, which implements a distributed computations of $H_{1}(\mathcal{R})$. We also note that besides the coverage problem discussed in this paper, $H_{1}(\mathcal{R})$ also plays important role to repair holes in the covering as discussed in [3].

This paper is organized as follows. In section 2, we prove a proposition, which is necessary for the construction of our algorithm via Mayer-Vietoris sequences. We show several equivalent sufficient conditions for the coverage of a non-pinching domain $D$ in section 3 . Section 4 is the main part of this paper and is devoted to present the distributed $H_{1}(\mathcal{R})$ computations, which enable us to distribute the computations for checking a sufficient condition of the coverage. In the last section, we discuss the computational cost and the further extensions of our algorithms.

\section{Mathematical preliminary}

The purpose of this section is to prove the following proposition. 
Proposition 3 Let $\mathcal{R}_{1}, \mathcal{R}_{2}$ be Rips complexes satisfying

$$
H_{0}\left(\mathcal{R}_{1}\right) \cong H_{0}\left(\mathcal{R}_{2}\right) \cong \mathbb{Z}, H_{0}\left(\mathcal{R}_{1} \cap \mathcal{R}_{2}\right) \cong \mathbb{Z}^{r}, H_{1}\left(\mathcal{R}_{1}\right) \cong \mathbb{Z}^{n}, H_{1}\left(\mathcal{R}_{2}\right) \cong \mathbb{Z}^{m}
$$

Then the first homology group of $\mathcal{R}_{1} \cup \mathcal{R}_{2}$ is given by

$$
H_{1}\left(\mathcal{R}_{1} \cup \mathcal{R}_{2}\right) \cong \begin{cases}\mathbb{Z}^{n+m} & (r=0) \\ \mathbb{Z}^{n+m-L+r-1} & (r \geq 1)\end{cases}
$$

where $L$ denotes the rank of the homomorphism $i_{1}$ that appears in the MayerVietoris sequence

$$
\cdots \longrightarrow H_{1}\left(\mathcal{R}_{1} \cap \mathcal{R}_{2}\right) \stackrel{i_{1}}{\longrightarrow} H_{1}\left(\mathcal{R}_{1}\right) \oplus H_{1}\left(\mathcal{R}_{2}\right) \longrightarrow H_{1}\left(\mathcal{R}_{1} \cup \mathcal{R}_{2}\right) \longrightarrow \cdots
$$

Proof. We consider the Mayer-Vietoris sequence for $\mathcal{R}_{1}$ and $\mathcal{R}_{2}$

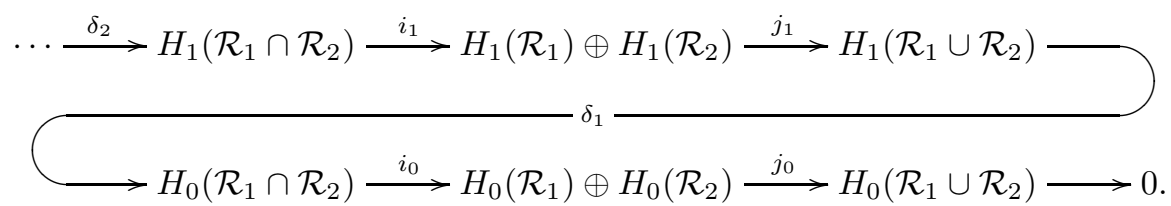

The short exact sequence

$$
0 \longrightarrow \operatorname{Im} j_{1} \longrightarrow H_{1}\left(\mathcal{R}_{1} \cup \mathcal{R}_{2}\right) \stackrel{\delta_{1}}{\longrightarrow} \operatorname{Im} \delta_{1} \longrightarrow 0
$$

splits since $\operatorname{Im} \delta_{1}$, being a subgroup of $H_{0}\left(\mathcal{R}_{1} \cap \mathcal{R}_{2}\right) \cong \mathbb{Z}^{r}$, is free. Therefore we have

$$
H_{1}\left(\mathcal{R}_{1} \cup \mathcal{R}_{2}\right) \cong \operatorname{Im} j_{1} \oplus \operatorname{Im} \delta_{1} .
$$

By virtue of Theorem $2, H_{1}\left(\mathcal{R}_{1} \cup \mathcal{R}_{2}\right)$ is free and hence so is $\operatorname{Im} j_{1}$. Thus, for computing $\operatorname{Im} j_{1}$, it suffices to know the rank of $i_{1}$. That is, we have

$$
\operatorname{Im} j_{1} \cong\left(H_{1}\left(\mathcal{R}_{1}\right) \oplus H_{1}\left(\mathcal{R}_{2}\right)\right) / \operatorname{Ker} j_{1} \cong \mathbb{Z}^{n+m} / \operatorname{Im} i_{1} \cong \mathbb{Z}^{n+m-L} .
$$

Next, we compute $\operatorname{Im} \delta_{1}$, which depends on $r$. If $r=0$ then $\mathcal{R}_{1} \cap \mathcal{R}_{2}$ is empty and hence $H_{1}\left(\mathcal{R}_{1} \cap \mathcal{R}_{2}\right)$ also vanishes. It follows from the Mayer-Vietoris sequence that $H_{1}\left(\mathcal{R}_{1} \cup \mathcal{R}_{2}\right) \cong \mathbb{Z}^{n+m}$. Assume $r \geq 1$. Since $H_{0}\left(\mathcal{R}_{1} \cap \mathcal{R}_{2}\right)$ is nontrivial, $\mathcal{R}_{1}$ and $\mathcal{R}_{2}$ must have at least one 0 -simplex in common, and therefore it follows from $H_{0}\left(\mathcal{R}_{1}\right) \cong H_{0}\left(\mathcal{R}_{2}\right) \cong \mathbb{Z}$ that $H_{0}\left(\mathcal{R}_{1} \cup \mathcal{R}_{2}\right) \cong \mathbb{Z}$. Therefore, the bottom row of the Mayer-Vietoris sequence induces

$$
0 \longrightarrow \operatorname{Im} \delta_{1} \longrightarrow \mathbb{Z}^{r} \stackrel{i_{0}}{\longrightarrow} \mathbb{Z}^{2} \stackrel{j_{0}}{\longrightarrow} \mathbb{Z} \longrightarrow 0
$$

which implies $\operatorname{Im} \delta_{1} \cong \mathbb{Z}^{r-1}$.

We note that in view of Theorem 2, the assumptions on the first homology groups $H_{1}$ in Proposition 3 is not restrictive at all. 


\section{Sufficient conditions for the coverage}

Since the first homology group $H_{1}(\mathcal{R})$ of the Rips complex $\mathcal{R}$ is the same as $H_{1}(S)$ by Theorem 2 , intuitively saying, the rank of $H_{1}(\mathcal{R})$ counts the number of "holes" in the shadow $S$. However, this hole may be outside the region $D$ and hence unrelated to the coverage problem. This happens when there exists a path between fence nodes which runs off the region $D$. To avoid this ambiguity, we add one assumption on the locations of nodes, which guarantees "nonpinching" via the Rips shadow around the boundary as shown in Figure 1.

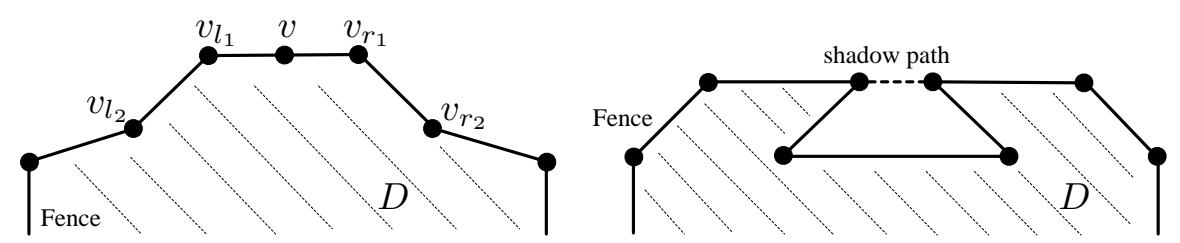

Fig. 1 The left figure shows a non-pinching situation and the right figure shows a pinching situation, respectively.

Assumption A5. The Rips shadow $S$ is included in $D$.

Note that $\mathbf{A} 5$ holds automatically if the region $D$ is convex, that is, for all $x$ and $y$ in $D$ and all $t \in[0,1]$, we have $(1-t) x+t y \in D$. If this is not the case, A5 might be difficult to check from the local information of each sensor. However, the following verifiable mild condition may be sufficient in practice from the engineering viewpoint.

For each fence node $v$, let us denote its two neighboring fence nodes on both sides as $v_{l_{1}}, v_{l_{2}}, v_{r_{1}}, v_{r_{2}}$. A non-pinching condition for $v$ is introduced in such a way that all fence nodes except for $v_{l_{1}}, v_{l_{2}}, v_{r_{1}}, v_{r_{2}}$ do not have edges to $v$ (See Figure 1). Then A5 can be replaced by a verifiable condition:

Assumption $\widetilde{\mathbf{A 5}}$. All fence nodes satisfy the non-pinching condition.

Rigorously speaking, it is possible to show exceptional examples like in Figure 2 such that $\widetilde{\mathbf{A 5}}$ does not induce A5. The reason for the existence of such examples is related to the definition of the fence nodes, but we can remove these singular situations by $\widetilde{\mathbf{A 5}}$ with slight modifications of fence nodes, e.g., dealing with $v$ as a fence node in Figure 2. Another example of the exception can be considered as depicted in Figure 3. However, we can ignore the exception as we will see in the end of this section. Therefore, for the practical purposes, $\widetilde{\mathbf{A 5}}$ is enough to guarantee A5.

Then, the sufficient condition for coverage in Theorem 1 can be described in the following equivalent forms.

Proposition 4 Under the assumption A1-A5, the following four conditions are equivalent: 


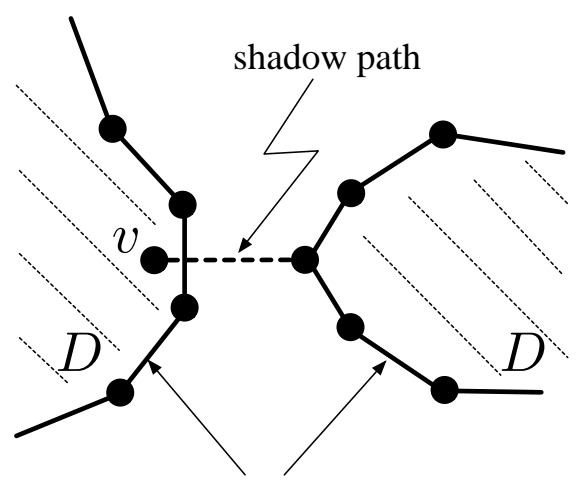

Fence

Fig. 2 An exceptional example satisfying $\widetilde{\mathbf{A 5}}$, but not $\mathbf{A 5}$.

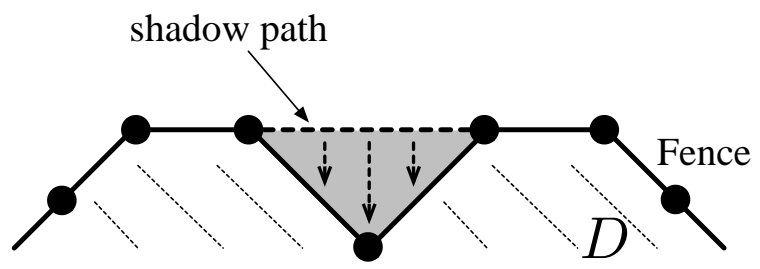

Fig. 3 Another exceptional example. But this shadow path can be homotopically deformable to a portion of $\partial D$ via a 2 -simplex. Hence, Proposition 4 is still valid in this case.

1. There exists $[\alpha] \in H_{2}(\mathcal{R}, \mathcal{F})$ such that $\delta_{2}[\alpha] \neq 0$ in $H_{1}(\mathcal{F})$.

2. The homomorphism $j_{1}: H_{1}(\mathcal{R}) \rightarrow H_{1}(\mathcal{R}, \mathcal{F})$ is an isomorphism.

3. The inclusion map $i_{1}: H_{1}(\mathcal{F}) \rightarrow H_{1}(\mathcal{R})$ is trivial. That is, $i_{1}=0$.

4. $H_{1}(\mathcal{R})=0$.

Here $j_{1}$ and $i_{1}$ are homomorphisms in the long exact sequence of the pair $(\mathcal{R}, \mathcal{F})$

$\cdots \longrightarrow H_{2}(\mathcal{R}, \mathcal{F}) \stackrel{\delta_{2}}{\longrightarrow} H_{1}(\mathcal{F}) \stackrel{i_{1}}{\longrightarrow} H_{1}(\mathcal{R}) \stackrel{j_{1}}{\longrightarrow} H_{1}(\mathcal{R}, \mathcal{F}) \stackrel{\delta_{1}}{\longrightarrow} H_{0}(\mathcal{F}) \stackrel{i_{0}}{\longrightarrow} \cdots$.

Proof The proofs for $2 \Rightarrow 3$ and $4 \Rightarrow 1$ are trivial from the exactness.

$(1 \Rightarrow 2)$ : Since $\operatorname{Im} \delta_{2}$ is a subgroup of $H_{1}(\mathcal{F}) \cong \mathbb{Z}$, it takes a form $\operatorname{Im} \delta_{2} \cong c \mathbb{Z}$. From the assumption, $c$ is a nonzero integer. Since

$$
H_{1}(\mathcal{F}) / \operatorname{Ker} i_{1} \cong H_{1}(\mathcal{F}) / \operatorname{Im} \delta_{2} \cong \mathbb{Z} / c \mathbb{Z} \cong \mathbb{Z}_{c}
$$

and $H_{1}(\mathcal{R})$ is free, the integer $c$ must be one. This leads to $i_{1}=0$, and so $j_{1}$ is injective. In addition, Ker $i_{0}=0$ results in $\delta_{1}=0$, so implies the surjectivity of $j_{1}$. This leads to the statement 2 .

$(3 \Rightarrow 4)$ : First, note that $S \subset D$ holds from A5. To prove $H_{1}(\mathcal{R})=0$, it 
suffices to show that $S=D$. Because, this implies $\pi_{1}(S)$ is trivial and then it follows from Theorem 2 that so is $\pi_{1}(R)$.

By contradiction, assume that $S \neq D$. Then $H_{1}(S)$ is a non-trivial group generated by loops around the components of $D \backslash S$. In particular, the shadow of the fence cycle is non-zero in $H_{1}(S)$ since $p(\mathcal{F})=\partial D$.

Now, consider the following diagram:

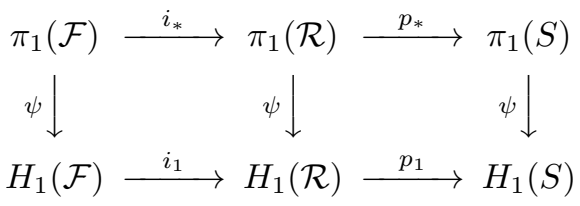

where $\psi$ is the Hurewicz homomorphism. The functorial property of $\psi$ implies that this diagram commutes. Regard the fence cycle $\mathcal{F}$ as an element of $\pi_{1}(\mathcal{F})$. Then

$$
\psi \circ p_{*} \circ i_{*}(\mathcal{F})=p_{1} \circ \psi \circ i_{*}(\mathcal{F}) \neq 0
$$

since it corresponds to the shadow of the fence cycle in $H_{1}(S)$. It follows that

$$
\psi \circ i_{*}(\mathcal{F})=i_{1} \circ \psi(\mathcal{F}) \neq 0 \text {. }
$$

This contradicts our assumption $i_{1}=0$.

It should be noted that the condition A5 is necessary only for the proof $(3 \Rightarrow 4)$. This proof shows that another exceptional case that $\widetilde{\mathbf{A 5}}$ does not induce A5 like Figure 3 is allowed for the validity of Proposition 4. This is because a shadow edge outside of $D$ can be now homotopically deformable to some portions of $\partial D$. In this sense, the condition $\widetilde{\mathbf{A 5}}$, with slight modifications concerning fence nodes if necessary, is suitable for practical implementations. The reason to add $\mathbf{A 5}$ or $\widetilde{\mathbf{A 5}}$ is related to distributed computations which will be proposed in the next section. We will explain this subject in section 5 in more detail.

Now we have reached the goal of this section. By combining Proposition 4 with Theorem 1, we obtain the following sufficient condition for the coverage.

Corollary 5 Under the assumptions A1-A5, sensors cover $D$ if $H_{1}(\mathcal{R})=0$.

\section{Distributed computation}

In this section, we present two algorithms for the distributed computation of the coverage condition under A1-A5 (or $\widetilde{\mathbf{A 5}}$ ). For this purpose, let us distinguish the first $K$ sensors as core sensors and, to each core sensor $v_{k}(k=$ $1, \cdots, K)$, assign the primary ID $k$. All calculation related homology computations will be carried out on the core sensors. We assume that core sensors can communicate the data of homology groups and matrices used in the computation of homology groups with each other. 
The essence of the following algorithm is divided into two processes. (1) Spreading the primary ID to adjacent nodes which have not yet obtained the primary ID based on the breadth first search. The adjacent nodes with different primary IDs mutually share their primary IDs as their sub IDs. (2) Sensors on the leaf of the breadth first search tree compose " $k$-connection sheet" and return it to their parents. Here a $k$-connection sheet consists of the following two lists:

(1) A list of edges connecting two sensors both of which have $k$ as the primary ID or a sub ID number.

(2) A list of the primary ID and all sub ID numbers for each sensor appearing in the list (1).

Algorithm 6 (Decomposition of Rips Complexes) Repeat the following procedure until all core sensors obtain their connection sheets.

1. Each sensor (say $v$ ) which already holds own primary ID (say $k$ ) performs the following (a), (b), (c) and (d) in order:

(a) If all adjacent sensors of $v$ have been already assigned a primary ID or have already been assigned $k$ as one of their sub IDs, then $v$ proceeds to process 2.

(b) The sensor $v$ assigns $k$ as the primary ID to its adjacent sensors whose primary IDs have not been assigned yet. The sensors which obtained $k$ as their primary ID here (primary children of $v$ ) start process 1 (we call $v$ the primary parent of these sensors).

(c) The sensor $v$ assigns $k$ as sub IDs to its adjacent sensors which have been already assigned the primary ID other than $k$ and does not have been assigned $k$ as a sub ID. The sensors which obtained $k$ as their sub IDs here ( $k$-sub children of $v$ ) proceed to process 3 (we call $v$ the $k$-sub parent of these sensors).

(d) The sensor $v$ waits for its primary children and $k$-sub children to return their $k$-connections sheets. When all answers arrive, $v$ proceeds to process 4 .

2. The sensor $v$ with primary ID $k$ returns to its primary parent the following data as the $k$-connection sheet: (1) the edge between $v$ and its primary parent; (2) its primary ID $k$. Then $v$ stops (waiting possible assignments of $s u b$ IDs).

3. The sensor $v$ obtained a sub ID $k$ waits for all its adjacent sensors to obtain their primary IDs (and therefore $v$ obtains all its sub IDs) and then return the following data to its $k$-sub parent as the $k$-connection sheet: (1) the list of edges between $v$ and sensors having $k$ as primary or sub ID; (2) the list of primary and sub IDs of $v$. Then $v$ stops.

4. The sensor $v$ collects all $k$-connection sheets from its primary and $k$-sub children. Combine these sheets into a single $k$-connection sheet and add the following data to it: (1) the list of edges between $v$ and sensors having $k$ as primary or sub ID; (2) the list of primary and sub IDs of $v$. By combining and adding we mean taking the union of lists. Then $v$ returns the $k$-connection sheet to its primary parent and stops. 
Let us remark that sensors may have multiple sub IDs and the process of 3 and 4 in the algorithm above are executed for each sub ID in parallel. All sensors appearing in the $k$-connection sheet of the core sensor $v_{k}$ must possess the primary or sub ID number $k$, and vice versa.

We note that the $k$-connection sheet defines a graph $G_{k}$ by the list of edges. Moreover, let us define a subgraph $G_{k l}$ by the list of all edges in the $k$-connection sheet whose nodes on both sides have the primary or sub ID of $l$. It is obvious that $G_{k l}=G_{l k}$. Let us denote by $\mathcal{R}_{k}$ and $\mathcal{R}_{k l}$ the Rips complexes determined by the graph $G_{k}$ and $G_{k l}$, respectively. Then, the following proposition about Algorithm 6 holds.

\section{Proposition 7}

1. Algorithm 6 finishes in finite steps.

2. If the Rips complex $\mathcal{R}$ is connected, all nodes obtain their unique primary IDs.

3. If the Rips complex $\mathcal{R}$ is connected, $\mathcal{R}=\cup_{k=1}^{K} \mathcal{R}_{k}$.

4. $\mathcal{R}_{k} \cap \mathcal{R}_{l}=\mathcal{R}_{k l}$.

Proof The statements 1, 2, 4 and $\mathcal{R} \supset \cup_{k=1}^{K} \mathcal{R}_{k}$ are clear. Suppose $\sigma \in \mathcal{R}$. Let us assume there exist two nodes $v_{a}$ and $v_{b}$ in $\sigma$ holding different primary IDs $k_{a}$ and $k_{b}$, respectively. Since $v_{b}$ is an adjacent node of $v_{a}$, it has a sub ID $k_{a}$. Therefore, all nodes in $\sigma$ are included in $\mathcal{R}_{a}$, and $\sigma \in \mathcal{R}_{a}$ holds.
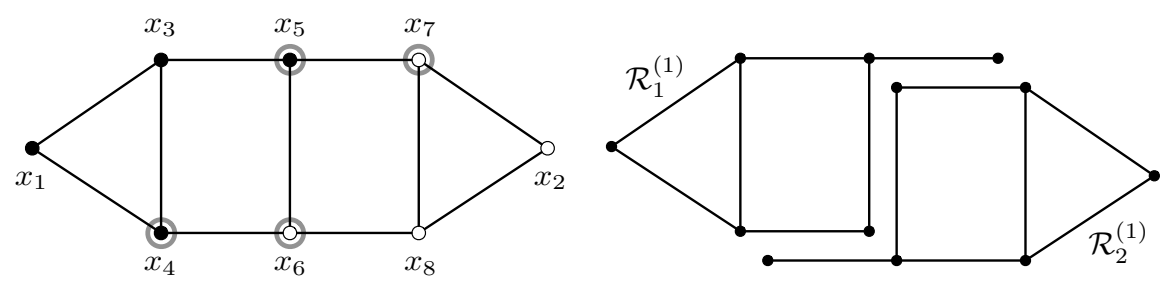

Fig. 4 The left figure shows assignments of primary and sub IDs to the communication network, where $x_{1}$ and $x_{2}$ are core sensors. Black nodes $\left(x_{1}, x_{3}, x_{4}\right.$ and $\left.x_{5}\right)$ obtain primary ID 1 while white nodes $\left(x_{2}, x_{6}, x_{7}\right.$ and $\left.x_{8}\right)$ obtain primary ID 2 . Nodes with gray circles obtain sub IDs. Namely, $x_{4}$ and $x_{5}$ obtain sub ID 2 , while $x_{6}$ and $x_{7}$ obtain sub ID 1 . The right figure shows the derived 1-skeletons of $\mathcal{R}_{1}$ and $\mathcal{R}_{2}$.

We remark that sub IDs possess information about intersections of the Rips subcomplexes $\mathcal{R}_{k}, k=1, \cdots, K$. It is obvious that, if the Rips complex is not connected, then allocating one core sensor at least to each connected component guarantees the same results of the statements 2 and 3 in Proposition 7. In Figure 4, we describe an example of assignments of primary and sub IDs in the case of $K=2$, where $x_{1}$ and $x_{2}$ are core sensors. The corresponding 1 and 2-connection sheets are shown in Table 1.

Now, we are in the position to present an algorithm for distributed $H_{1}(\mathcal{R})$ computations by Proposition 3 and Algorithm 6 . 


\begin{tabular}{|c|c|c|c|}
\hline \multicolumn{2}{|c|}{ 1-connection sheet } & \multicolumn{2}{|c|}{ 2-connection sheet } \\
\hline Edge list & ID list & Edge list & ID list \\
\hline$(1,3)$ & $x_{1}:(1)$ & $(2,7)$ & $x_{2}:(2)$ \\
\hline$(1,4)$ & $x_{3}:(1)$ & $(2,8)$ & $x_{4}:(1,2)$ \\
\hline$(3,4)$ & $x_{4}:(1,2)$ & $(4,6)$ & $x_{5}:(1,2)$ \\
\hline$(3,5)$ & $x_{5}:(1,2)$ & $(5,6)$ & $x_{6}:(2,1)$ \\
\hline$(4,6)$ & $x_{6}:(2,1)$ & $(5,7)$ & $x_{7}:(2,1)$ \\
\hline$(5,6)$ & $x_{7}:(2,1)$ & $(6,8)$ & $x_{8}:(2)$ \\
\hline$(5,7)$ & & $(7,8)$ & \\
\hline
\end{tabular}

Table 11 and 2-connection sheets of $\mathcal{R}_{1}$ and $\mathcal{R}_{2}$ in Figure 4, respectively. In the edge list, each pair of numbers expresses the edge with the corresponding vertices. In the ID list, the first number shows the primary ID and the next shows the sub ID.

\section{Algorithm 8 (Distributed $H_{1}(\mathcal{R})$ computations)}

1. Decompose the Rips complex $\mathcal{R}$ into Rips subcomplexes $\left\{\mathcal{R}_{k} \mid k=1, \cdots, K\right\}$ by Algorithm 6.

2. Each core sensor $v_{k}(k=1, \ldots, K)$ computes the homology group $H_{1}\left(\mathcal{R}_{k}\right)$ of corresponding Rips subcomplex $\mathcal{R}_{k}$.

3. Let $k=2$.

4. The core sensor $v_{1}$ calculates $H_{1}\left(\mathcal{R}_{1} \cup \mathcal{R}_{k}\right)$ by means of Proposition 3.

5. Change primary and sub ID numbers $k$ to 1 in all connection sheets.

6. Replace $k$ with $k+1$ and repeat the processes 4 and 5 until $k=K$.

By this algorithm, the homology group $H_{1}(\mathcal{R})$ is calculated by summing up the homology groups $H_{1}\left(\mathcal{R}_{k}\right)$.

A standard algorithm using the Smith normal form (see Chapter 3 of [8], or Section 1.13 of [11]) can be applied for the computation of the homology groups. Here we briefly recall the algorithm. Assume we want to compute the homology group of a Rips complex $\mathcal{R}$. Denote by $C_{n}$ the $n$-chain group of $\mathcal{R}$, the group consists of formal linear combinations of the form $m_{1} \sigma_{1}+\cdots+m_{l} \sigma_{l}$ where $m_{i}$ are integers and $\sigma_{i}$ are $n$-simplices. We denote by $A_{n}$ the matrix representing the boundary map $\partial_{n}: C_{n} \rightarrow C_{n-1}$ with respect to this basis of $C_{n}$ and $C_{n-1}$. What the homology computation algorithm does is to construct nice bases of $C_{n}$ so that the matrices representing the boundary maps take the form of rectangular diagonal (not necessary square) matrices; that is, it composes elementary transformations to form unimodular matrices $M_{n}, N_{n}$ : $C_{n} \rightarrow C_{n}$ such that $M_{n}$ and $N_{n}$ are inverse to each other and the product

$$
D_{n}:=N_{n-1} A_{n} M_{n},
$$

which represents $\partial_{n}$ in the new bases, is rectangular diagonal for $n=0,1, \ldots$, $\operatorname{dim} \mathcal{R}$ (The matrix $N_{-1}$ is defined to be the identity). For simplicity, we call this new basis of $C_{n}$ the homological basis, and the original basis of $C_{n}$ the simplicial basis. With homological bases, we can easily compute $H_{n}(\mathcal{R})=$ Ker $\partial_{n} / \operatorname{Im} \partial_{n+1}$. Namely,

$$
H_{n}(\mathcal{R})=\bigoplus_{j} \mathbb{Z} / d_{i} \mathbb{Z}
$$


where the sum is taken over all $j$ such that $j$-th diagonal element of $D_{n}$ is 0 and $d_{j}$ is the $j$-th diagonal element of $D_{n+1}$. Remark that if $\mathcal{R}$ is a planner Rips complex, then its first homology group is free and therefore we have

$$
H_{1}(\mathcal{R})=\bigoplus_{j} \mathbb{Z}
$$

where the sum is taken over all $j$ such that $j$-th diagonal element of $D_{n}$ and $D_{n+1}$ are both 0 . That is, the basis of $H_{1}(\mathcal{R})$ is obtained as a subset of the homological basis of $C_{1}$.

The essential part of the algorithm is process 4 . Here the core sensor $v_{1}$ is required to compute the rank $r$ of $H_{0}\left(\mathcal{R}_{1} \cap \mathcal{R}_{k}\right)$ and the rank $L$ of the homomorphism

$$
i_{1}: H_{1}\left(\mathcal{R}_{1} \cap \mathcal{R}_{k}\right) \rightarrow H_{1}\left(\mathcal{R}_{1}\right) \oplus H_{1}\left(\mathcal{R}_{k}\right)
$$

The rank $r$ can also be computed by the standard homology computation algorithm.

Now we discuss how to compute $L$, the rank of $i_{1}$. We first construct the matrix representing $i_{1}$. Since $H_{1}\left(\mathcal{R}_{1} \cap \mathcal{R}_{k}\right), H_{1}\left(\mathcal{R}_{1}\right)$ and $H_{1}\left(\mathcal{R}_{k}\right)$ are free, there exist bases $\alpha_{1}, \ldots, \alpha_{a}$ of $H_{1}\left(\mathcal{R}_{1} \cap \mathcal{R}_{k}\right), \beta_{1}, \ldots, \beta_{b}$ of $H_{1}\left(\mathcal{R}_{1}\right)$ and $\gamma_{1}, \ldots, \gamma_{c}$ of $H_{1}\left(\mathcal{R}_{k}\right)$. Here $a, b$ and $c$ are the ranks of corresponding homology groups. The matrix will be constructed with respect to these bases. Let $\alpha$ be one of $\alpha_{1}, \ldots, \alpha_{a}$. We will see how to determine its image $i_{1}(\alpha)$ in $H_{1}\left(\mathcal{R}_{1}\right)$. The image in $H_{1}\left(\mathcal{R}_{k}\right)$ can be determined in the same way. Denote by $M_{n}^{(1, k)}$ and $N_{n}^{(1, k)}$ the unimodular matrices constructed in the computation of $H_{1}\left(\mathcal{R}_{1} \cap \mathcal{R}_{k}\right)$, and by $D_{n}^{(1, k)}$ the diagonal representation of the $n$-th boundary map. Similarly, we denote by $M_{n}^{(1)}, N_{n}^{(1)}$ and $D_{n}^{(1)}$ these matrices with respect to $H_{1}\left(\mathcal{R}_{k}\right)$. We also denote by $C_{n}\left(\mathcal{R}_{1} \cap \mathcal{R}_{k}\right)$ and $C_{n}\left(\mathcal{R}_{1}\right)$ the $n$-chains of $\mathcal{R}_{1} \cap \mathcal{R}_{k}$ and $\mathcal{R}_{1}$. Assume $\alpha$ corresponds to the $j$-th coordinate vector $e_{j}:={ }^{t}(0, \ldots, 0,1,0, \ldots, 0)$, where 1 appears only in the $j$-th row, of $C_{1}\left(\mathcal{R}_{1} \cap \mathcal{R}_{k}\right)$ with respect to the homological basis. Then in the simplicial basis of $C_{1}\left(\mathcal{R}_{1} \cap \mathcal{R}_{k}\right), \alpha$ is represented by $M_{1}^{(1, k)} e_{j}$. Since a simplex of $\mathcal{R}_{1} \cap \mathcal{R}_{k}$ is also a simplex of $\mathcal{R}_{1}$, we can naturally embed $C_{1}\left(\mathcal{R}_{1} \cap \mathcal{R}_{k}\right)$ into $C_{1}\left(\mathcal{R}_{1}\right)$. Let $E$ be the matrix representing this embedding. Then $E M_{1}^{(1, k)} e_{j}$ is the vector which represents the chain $\alpha$ in terms of the simplicial basis of $C_{1}\left(\mathcal{R}_{1}\right)$. To take homology, we first change the coordinate from simplicial to homological by multiplying $N_{1}^{(1)}$, obtaining $N_{1}^{(1)} E M_{1}^{(1, k)} e_{j}$. The projection of $C_{1}\left(\mathcal{R}_{1}\right)$ onto $H_{1}\left(\mathcal{R}_{1}\right)$ can be easily computed in the homological basis, by simply omitting rows not corresponding to $\beta_{1}, \ldots, \beta_{b}$, the basis of $H_{1}\left(\mathcal{R}_{1}\right)$. The result is exactly the vector representing $i_{1}(\alpha)$ with respect to $\beta_{1}, \ldots, \beta_{b}$. Thus we have determined the first column of the matrix representing $i_{1}$. We determine the other columns in the same way. Finally, to compute the rank of $i_{1}$, it suffices to construct the Smith normal form of this matrix.

Recall that from Proposition 4 the sufficient condition for the coverage under the assumption A1-A5 (or $\widetilde{\mathbf{A 5}}$ ) can be expressed as $H_{1}(\mathcal{R})=0$. There- 
fore this algorithm enables distributed computations of the coverage condition, which will be indispensable for practical implementations.

We remark that the above procedure for summing up the homology groups $H_{1}\left(\mathcal{R}_{k}\right)$ is a very primitive one. It causes computational unbalance focusing on the core sensor $v_{1}$. In order to avoid this problem, a simple modification should be taken into account. For example, we may adopt distributed computations in Algorithm 8 in such a way that some preassigned core sensors execute processes 4 and 5 simultaneously with their nearby core sensors.

\section{Discussions}

In this paper, we have discussed a coverage problem and presented an algorithm to check the sufficient condition for the coverage expressed by means of homology groups in a distributed manner. We here discuss its computational costs and further extensions.

First of all, let us consider the total computational cost of Algorithm 8 . Obviously, homology computations determine the total computational complexity.

Most homology computations are based on the Smith normal forms and, in general, need polynomial orders with respect to the number of simplices (see [8] or [13] for the recent development of homology computations). The order $p$ of polynomials depends on geometric settings of problems in the range $2 \leq p \leq 5$.

In the homology computations in Algorithm 8, we need to calculate $H_{1}\left(\mathcal{R}_{k}\right)$ and $H_{1}\left(\mathcal{R}_{1} \cap \mathcal{R}_{k}\right)$ for $k=1, \cdots, K$ and the rank $L$ for each summing process. It should be noted that we can apply the method discussed in the paper [2] to speed up the computations of rank $L$. This method enables us to detect whether a cycle with $k$ edges is contractible in our settings and its computational cost is $O(k)$ with $O(m \log n)$ preprocess, where $m$ and $n$ are the numbers of edges and points in the Rips complex. That is, the testing of contractibility is faster than homology computations. At the process 4 of Algorithm 8 , therefore, we may apply this contractibility test for each basis of $H_{1}\left(\mathcal{R}_{1} \cap \mathcal{R}_{k}\right)$ before computing its image under $i_{1}$. If it is contractible in $\mathcal{R}_{1}$ (or $\mathcal{R}_{k}$ ), we can safely conclude that the image is 0 in $H_{1}\left(\mathcal{R}_{1}\right)$ (or in $H_{1}\left(\mathcal{R}_{k}\right)$ ). We can also reduce the computational cost by using $\mathbb{Z}_{2}$ instead of $\mathbb{Z}$ as the coefficient of homology groups. No information would be lost through this reduction since the first homology groups of the Rips complexes are free (Theorem 2). The more detailed estimates with respect to computation-communication trade-offs are future problems.

Next, let us discuss the extensions of the presented distributed computations. One extension of the algorithm is to construct distributed computation algorithms also for homology groups except for $H_{1}(\mathcal{R})$. It should be noted that the equivalence of the sufficient conditions 1 and 2 under A1-A4 in Proposition 4 is easily checked. However, the reason that we have to put the assumption $\mathbf{A 5}$ or $\widetilde{\mathbf{A 5}}$ for our settings is to avoid computations of $H_{1}(\mathcal{R}, \mathcal{F})$ 
and the homomorphism $j_{1}$. Therefore, further extensions of distributed computations to other homology groups may remove the non-pinching condition A5 or $\widetilde{\mathbf{A 5}}$.

Finally, we would like to mention the three dimensional coverage problem [3]. In this case, the corresponding Rips complex is derived from a set of finite points in $\mathbb{R}^{3}$. In order to apply our theory, we need to clarify the topological properties of these Rips complexes, as Chambers et al. did for the two dimensional case [1]. This is a challenging problem from both engineering and mathematical viewpoints.

Acknowledgements The authors express their sincere gratitude to Dr. Nobuyoshi Takahashi for useful comments on Theorem 2. They thank the members of TIN working group, especially Professors Shinsuke Hara and Hiroshi Kokubu, for stimulating discussions. They would also like to thank the anonymous reviewers for their careful reading and valuable suggestions.

\section{References}

1. E. Chambers, V. de Silva, J. Erickson, and R. Ghrist, Vietoris-Rips complexes of planar point sets, Discrete Comput. Geom. 44 (2010), 75-90.

2. E. W. Chambers, J. Erickson, and P. Worah, Testing contractibility in planar Rips complexes, Computational geometry (SCG'08), ACM, New York (2008), 251-259.

3. V. de Silva and R. Ghrist, Coordinate-free coverage in sensor networks with controlled boundaries via homology, Intl. J. Robotics Research 25 (2006), 1205-1222.

4. V. de Silva and R. Ghrist, Coverage in sensor networks via persistent homology, Alg. and Geom. Topology 7 (2007), 339-358.

5. R. Ghrist and A. Muhammad, Coverage and hole detection in sensor networks via homology, Proc. Information Processing in Sensor Networks (2005), 254-260.

6. M. Gromov, Hyperbolic groups, Essays in group theory, Mathematical Sciences Research Institute Publications 8, Springer-Verlag, New York, 1987, 75-263.

7. C.-F. Huang and Y.-C. Tseng, The coverage problem in a wireless sensor network, Proc. of the 2nd ACM international conference on wireless sensor networks and applications, ACM Press (2003), 115-121.

8. T. Kaczynski, K. Mischaikow, and M. Mrozek, Computational homology, Applied Mathematical Sciences 157, Springer-Verlag, New York, 2004.

9. H. Koskinen, On the coverage of a random sensor network in a bounded domain, Proceedings of 16th ITC Specialist Seminar (2004), 11-18.

10. X. Y. Li, P. J. Wan and O. Frieder, Coverage in wireless ad hoc sensor networks, IEEE Trans, on Computers 52 (2003), 753-763.

11. S. V. Matveev, Lectures on Algebraic Topology, EMS Series of Lectures in Mathematics, European Mathematical Society, Zurich, 2006.

12. S. Meguerdichian, F. Koushanfar, M. Potkonjak, and M. Srivastava, Coverage Problems in Wireless Ad-hoc Sensor Networks, IEEE INFOCOM (2001), 1380-1387.

13. M. Mrozek, Čech type approach to computing homology of maps, Discrete and Computational Geometry, DOI: 10.1007/s00454-010-9255-2, Springer, 2010.

14. E. Spanier, Algebraic topology, Springer-Verlag, 1966.

15. B. Srishnamachari, Networking wireless sensors, Cambridge University Press, 2005.

16. Yuh-Ren Tsai, Sensing coverage for randomly distributed wireless sensor networks in shadowed enyironments, IEEE Trans. on Vehicular Tech. 57 (2008), 556-564.

17. L. Vietoris, Über den höheren Zusammenhang kompakter Räume und eine Klasse von zusammenhangstreuen Abbildungen, Math. Ann. 97 (1927), 454-472.

18. P.-J. Wan and C.-W. Yi, Coverage by randomly deployed wireless sensor networks, IEEE Trans. on Information Theory, vol. 52 (2006), 2658-2669.

19. H. Zhang and J. C. Hou, Maintaining sensing coverage and connectivity in large sensor networks, Ad Hoc \& Sensor Wireless Networks (2005), 89-124. 